\title{
A Monte Carlo Background Covariance Localization Method for an Ensemble-Variational Assimilation System
}

\author{
IVo Pasmans AND AleXANDER L. KuRAPOV \\ College of Earth, Ocean, and Atmospheric Sciences, Oregon State University, Corvallis, Oregon
}

(Manuscript received 8 November 2016, in final form 5 September 2017)

\begin{abstract}
Spurious long-distance correlations in estimates of the background error covariance can deteriorate the performance of ensemble-based data assimilation methods. In this study, a localization method, called Monte Carlo (MC) localization, is presented to remove these correlations. It is particularly useful for use in highdimensional ensemble-variational data assimilation systems. In this method, raw ensemble members are truncated by multiplying them with functions having compact support. This creates a larger ensemble, in which points spaced farther apart than the size of the compact support have zero correlation. The localized background error covariance is then estimated as the sample covariance of this larger ensemble. It is hypothesized that this localized background error covariance can be approximated by the MC approximation method using a limited set of the truncated ensemble members. This hypothesis is tested here on a grid with 1001 grid points and assuming a Gaussian true background error covariance. It is found that the mean relative error has an upper bound that scales with the inverse square root of the number of truncated ensemble members. In the case studied the size of the support for which the localized background covariance best approximates the true background covariance increases with increasing number of raw ensemble members and is close to 4 times the standard deviation of the Gaussian when 20 raw ensemble members are used. In the Fourier space the localization manifests itself as a convolution resulting in smoothing of the power spectral density of the ensemble members.
\end{abstract}

\section{Introduction}

Operational implementations of four-dimensional variational data assimilation (4DVAR) in ocean models (Kurapov et al. 2011; Moore et al. 2011; Yu et al. 2012; Ngodock and Carrier 2014) require estimation of the covariance of the errors in the initial conditions prior to assimilation, or the background error covariance. The operational model that motivated our study is the Oregon-Washington coastal forecasting system (Oregon State University 2017). The configuration of this model is similar to that described in Kim et al. (2014). Its domain is approximately $400 \mathrm{~km}$ by $800 \mathrm{~km}$ with a $2-\mathrm{km}$ resolution in the horizontal and 40 terrain-following layers in the vertical. Its total dimensionality is of $O\left(10^{7}\right)$. The model assimilates sea surface temperature, altimeter sea surface height, and daily averaged surface current observations from high-frequency radars and uses a static background

\footnotetext{
Corresponding author: Ivo Pasmans, ipasmans@coas.oregonstate. edu
}

covariance (Kurapov et al. 2011). However, the background error statistics are expected to vary in space and time as result of changing environmental conditions and, in particular, because of the presence the Columbia River plume (Liu et al. 2009). To capture this variability, the background error covariance can be estimated from an ensemble of model runs.

A well-known problem with the estimation of the covariance from a limited number of ensemble members is the presence of spurious long-tail correlations that are detrimental to data assimilation performance (Hamill et al. 2001; Houtekamer and Mitchell 2001). To remove these correlations, various localization schemes have been proposed.

Many known localization schemes multiply the sample covariance on an element-by-element basis with a localized correlation matrix C (i.e., they use the Schur product). It is often assumed that correlations in $\mathbf{C}$ decrease with physical distance. In particular, piecewise rational functions with compact support (Gaspari and Cohn 1999) can be used to obtain sparse $\mathbf{C}$ (Houtekamer and Mitchell 2001; Buehner et al. 2010; Clayton et al. 
2013). Alternatively, ensemble members can be projected on a complete set of basis functions in spectral space, followed by localization of the correlation between the projection coefficients. A variety of choices for functions and localizations have been used. For example, in Derber and Bouttier (1999) and Berre (2000) horizontal Fourier basis functions are used and localization is performed by setting correlations between basis functions with different wavenumbers to zero. Buehner and Charron (2007) also localize in a spectral space spanned by the Fourier basis. However, they replace the Schur product in spectral space with convolution of the ensemble members with Gaspari and Cohn (1999) compact piecewise rational functions in physical space. In Deckmyn and Berre (2005), twodimensional (2D) Meyer wavelets are used as basis functions and the coefficients associated with different wavelets are set to zero. Combinations of the two aforementioned approaches have also been used: Buehner (2012) and Buehner and Shlyaeva (2015) bandpass filter the ensemble members and then apply spatial localization to each of the bandpass-filtered ensemble members using different localization scales for different bands. The disadvantage of these techniques is that they are nonadaptive: the matrix $\mathbf{C}$ does not depend on the model state. To overcome this disadvantage, Bishop and Hodyss (2007, 2009a,b) constructed $\mathbf{C}$ from the ensemble sample correlation by repeatedly taking the Schur product with itself. This reduces weak correlations stronger than strong correlations. To correct for the decrease of the strong correlations the result is smoothened. This adaptive method can be combined with the aforementioned nonadaptive methods (Bishop and Hodyss 2011). Anderson $(2007,2012)$ takes a different approach that does not require the construction of a localized correlation matrix C. Instead the data assimilative correction created by each observation is adjusted for sampling errors stemming from the use of an ensemble of limited size.

Not all of these localization schemes are suitable for implementation in our operational model. Transformation of ensemble members to/from spectral space is problematic near the irregularly shaped coast. For other schemes mentioned above implementation in an ensemble-variational model would be computationally expensive as they require the calculation of matrix-matrix products with matrices of dimensionality of $O\left(10^{7}\right)$ (Bishop and Hodyss 2009a), require the increase of the ensemble size (Anderson 2007), or application of $4 \mathrm{DVAR}$ to each of the $O\left(10^{4}\right)$ observations separately (Anderson 2007, 2012). For efficient implementation of nonadaptive localization in ensemble covariances in three-dimensional (3D) and 4D variational data assimilation schemes, Bishop et al. (2011) proposed (i) to perform the localization on a coarser grid, (ii) to use a localized correlation matrix in which the elements of the square root $\mathbf{C}^{1 / 2}$ are separable functions of horizontal and vertical coordinates, and (iii) assume that $\mathbf{C}^{1 / 2}$ is independent of the model variable localized. However, construction of $\mathbf{C}^{1 / 2}$ or the use of Gaspari and Cohn (1999) was found to be time consuming using computer resources available to us.

To reduce the time and computational cost involved in localization compared to Gaspari and Cohn (1999) and Bishop et al. (2011), we have constructed an alternative localization method, called Monte Carlo (MC) localization. This alternative method is described in detail in section 2 . In section 3 the computational cost of this method for a generic 3D model is compared to that of Bishop et al. (2011) and a method that uses $\mathbf{C}$ constructed using the piecewise fifth-order rational function from Gaspari and Cohn (1999). In section 4 we will look how well MC localization approximates the true background error covariance in a one-dimensional (1D) case with a Gaussian true covariance. Section 5 gives an example of MC localization implemented in a 3D oceanographic model. Section 6 discusses some of the drawbacks of MC localization and possible directions for improvement and contains a summary of our findings.

\section{Method}

In this paper we will assume that localization is applied in a finite-difference model on a structured grid with $N_{x}, N_{y}, N_{z}$ grid points in three directions $(x, y, z)$, respectively, that the spacing in each direction is equidistant, and that $N_{r}$ quantities are defined at each grid point. In this case the model state can be ordered as a vector of dimension $N=N_{x} N_{y} N_{z} N_{r}$. The support of a model state vector is defined as the set of grid points on which the elements in the vector can be nonzero.

By definition a correlation matrix C must be symmetric, nonnegative definite, and its diagonal elements must be equal to 1 . The first two requirements can be met by defining $\mathbf{C}$ as the sum of symmetric rank-1 matrices:

$$
\mathbf{C}=\sum_{m \in \mathbb{M}}\left(\boldsymbol{\chi}^{(m)} \circ \boldsymbol{\gamma}\right)\left(\boldsymbol{\chi}^{(m)} \circ \boldsymbol{\gamma}\right)^{\mathrm{T}}
$$

Here $\circ$ denotes the element-by-element multiplication of the Schur product, T is the matrix transpose, $\boldsymbol{\chi}^{(m)}$ are 
vectors of dimension $N$, while the indices $m$ are elements of some countable set $\mathbb{M} \subset \mathbb{N}$ of size $M$ (i.e., $m=1,2, \ldots, M)$. For the moment we only require the vectors $\boldsymbol{\chi}^{(m)}$ to be unique, but in the following $\boldsymbol{\chi}^{(m)}$ will be narrowed down to a specific class of vectors. Here $\gamma$ is a $N$-dimensional normalization vector that ensures that $\mathbf{C}$ has ones on its main diagonal. To achieve this, its elements must satisfy the following:

$$
\boldsymbol{\gamma}_{p}=\left[\sum_{m \in \mathbb{M}}\left(\boldsymbol{\chi}_{p}^{(m)}\right)^{2}\right]^{-1 / 2} .
$$

To make $\mathbf{C}$ a localization matrix, we add the requirement that $\mathbf{C}_{p q}=0$ if $d(p, q)$, the distance between points $p$ and $q$ in the metric $d$, is larger than a given localization distance. The distance between grid points $p$ and $q$ will be less than or equal to this localization distance if and only if there is a metric ball that has the localization distance as its diameter and contains both points $p$ and $q$. So, to satisfy the localization requirement, $\boldsymbol{\chi}^{(m)}$ must be a vector with its support in one of these balls.

Let $\left\{\mathbf{x}^{(k)}\right\}_{k}$, with $k=1,2, \ldots, K$, be an ensemble of $N$-dimensional model state vectors and let $\overline{\mathbf{x}}=$ $1 / K \sum_{k=1}^{K} \mathbf{x}^{(k)}$ be the ensemble mean. Then the raw, nonlocalized, ensemble sample covariance is

$$
\mathbf{B}_{\mathrm{ens}}=\frac{1}{K-1} \sum_{k=1}^{K}\left(\mathbf{x}^{(k)}-\overline{\mathbf{x}}\right)\left(\mathbf{x}^{(k)}-\overline{\mathbf{x}}\right)^{\mathrm{T}}
$$

Using the square root theorem [Bishop and Hodyss (2009b), their Eq. (1)] the localized estimate $\mathbf{B}_{\text {ens }} \circ \mathbf{C}$ for the background error covariance can be written as

$$
\begin{aligned}
\mathbf{B} & =\mathbf{B}_{\mathrm{ens}} \circ \mathbf{C} \\
& =\frac{1}{K-1} \sum_{k=1}^{K} \sum_{m \in \mathbb{M}}\left[\left(\mathbf{x}^{(k)}-\overline{\mathbf{x}}\right) \circ \boldsymbol{\chi}^{(m)} \circ \boldsymbol{\gamma}\right]\left[\left(\mathbf{x}^{(k)}-\overline{\mathbf{x}}\right) \circ \boldsymbol{\gamma}^{(m)} \circ \boldsymbol{\gamma}\right]^{\mathrm{T}}
\end{aligned}
$$

This is an $N \times N$-dimensional matrix of rank $M(K-1)$. Since (4) has the same structure as (3), the $N$-dimensional vectors $\left(\mathbf{x}^{(k)}-\overline{\mathbf{x}}\right) \circ \boldsymbol{\gamma}^{(m)} \circ \boldsymbol{\gamma}$ will be referred to as modulated ensemble members (Bishop and Hodyss 2011).

From now on we limit ourselves to $\boldsymbol{\chi}^{(m)}$ that are step functions on some ball. In particular, if $L_{x}, L_{y}, L_{z}$ are the localization distances in the $x, y, z$ directions, respectively, we define $\boldsymbol{\chi}^{(m)}$ as an $N$-dimensional vector with elements $\left(\boldsymbol{\chi}^{(m)}\right)_{p}=1$ if $L_{x}^{-1}\left|x_{p}-x_{m}\right|<1 / 2, L_{y}^{-1} \mid$ $y_{p}-y_{m}\left|<1 / 2, L_{z}^{-1}\right| z_{p}-z_{m} \mid<1 / 2$ and zero otherwise. In this way each grid point gives rise to one unique vector $\boldsymbol{\chi}^{(m)}$ and hence $M=N_{x} N_{y} N_{z}$ for this case. From here on
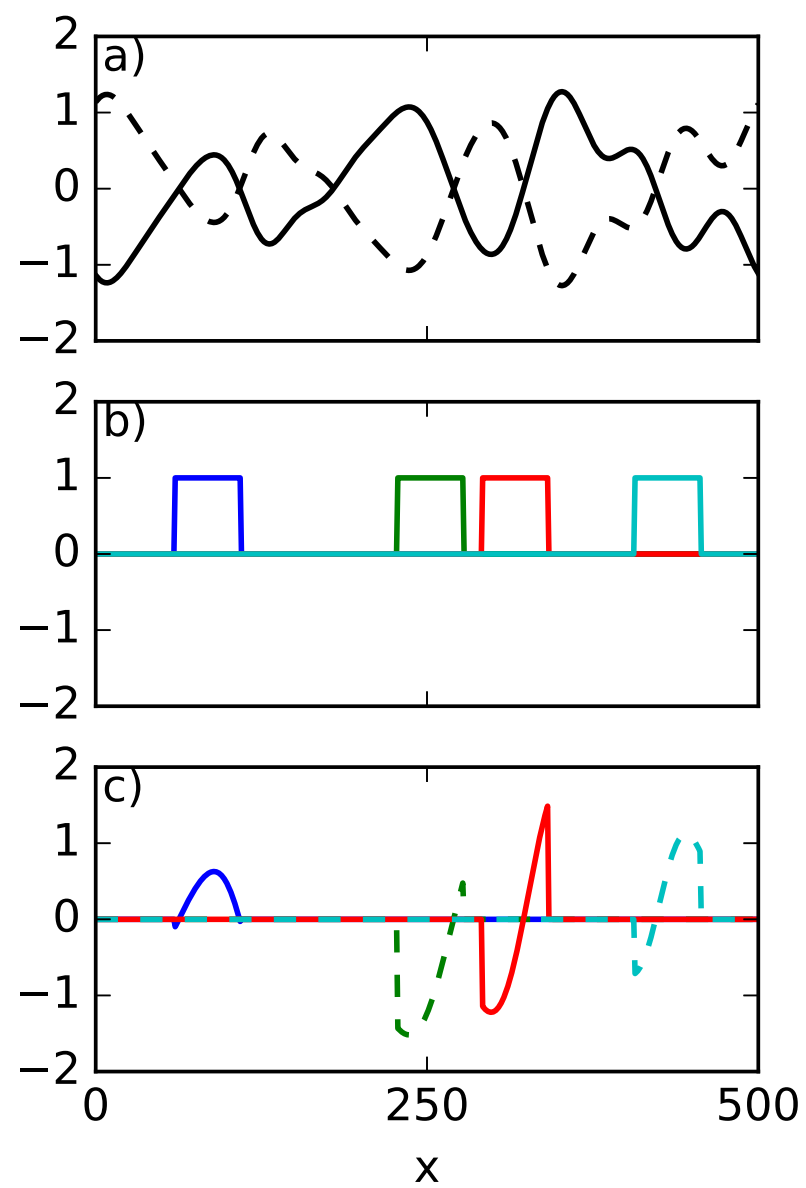

FIG. 1. 1D example showing the construction of the modulated ensemble members for the case $L_{\mathrm{MC}}=51$ on a 501 point grid. (a) Two raw ensemble members $\mathbf{x}^{(1)}-\overline{\mathbf{x}}$ (solid) and $\mathbf{x}^{(2)}-\overline{\mathbf{x}}$ (dashed). (b) Vectors $\boldsymbol{\chi}^{(85)}$ (dark blue), $\boldsymbol{\chi}^{(253)}$ (green), $\boldsymbol{\chi}^{(317)}$ (red), and $\chi^{(432)}$ (light blue). (c) The modulated ensemble members $\left(\mathbf{x}^{(1)}-\overline{\mathbf{x}}\right) \circ \boldsymbol{\chi}^{(85)} \circ \gamma($ dark blue $),\left(\mathbf{x}^{(2)}-\overline{\mathbf{x}}\right) \circ \boldsymbol{\chi}^{(253)} \circ \gamma($ green $)$, $\left(\mathbf{x}^{(1)}-\overline{\mathbf{x}}\right) \circ \boldsymbol{\chi}^{(317)} \circ \gamma($ red $)$, and $\left(\mathbf{x}^{(2)}-\overline{\mathbf{x}}\right) \circ \boldsymbol{\chi}^{(432)} \circ \gamma($ light blue $)$.

we will refer to the $\mathbf{B}$ constructed with all $K M$ vectors $\left[\left(\mathbf{x}^{(k)}-\overline{\mathbf{x}}\right) \circ \boldsymbol{\chi}^{(m)} \circ \boldsymbol{\gamma}\right]$ as $\mathbf{B}_{\text {full }}$.

An example of how a modulated ensemble can be constructed using this choice of vectors $\boldsymbol{\chi}^{(m)}$ is shown in Fig. 1. In this example there is only 1 model quantity, the model grid has only 1 spatial dimension, and it has 501 points with a grid spacing of $1\left(N=N_{x}=501\right)$. The compact support has a width of $L_{\mathrm{MC}}=51$ grid points. For two points that are farther than $L_{\mathrm{MC}}$ apart (e.g., point 340 and point 430 ), it is impossible to find a $\boldsymbol{\chi}^{(m)}$ in which both these points are nonzero. Consequently, a modulated ensemble member in which these points are both nonzero does not exist and hence $\left(\mathbf{B}_{\text {full }}\right)_{340,430}=0$ in this case.

The modulated ensemble is a factor $N_{x} N_{y} N_{z}$ larger than the raw ensemble. For our oceanographic model 
$N_{x} N_{y} N_{z} \approx O\left(10^{7}\right)$ and $K=50$ such that calculation of $\mathbf{B}_{\text {full }}$ is considerably more computationally intensive than that of $\boldsymbol{B}_{\text {ens. }}$. To speed up calculation of the localized ensemble covariance, we propose replacing (4) with its Monte Carlo (MC) approximation:

$$
\begin{aligned}
& \mathbf{B}_{\mathrm{MC}} \\
& \quad=\frac{1}{K-1} \sum_{k=1}^{K} \sum_{m \in \mathbb{M}_{k}}\left[\left(\mathbf{x}^{(k)}-\overline{\mathbf{x}}\right) \circ \boldsymbol{\chi}^{(m)} \circ \boldsymbol{\gamma}\right]\left[\left(\mathbf{x}^{(k)}-\overline{\mathbf{x}}\right) \circ \boldsymbol{\chi}^{(m)} \circ \boldsymbol{\gamma}\right]^{\mathrm{T}},
\end{aligned}
$$

where $\mathbb{M}_{k}$ is a randomly drawn subset of $\mathbb{M}=$ $\left\{1,2, \ldots, N_{x} N_{y} N_{z}\right\}$ of size $M_{\mathrm{MC}}$ and where $\mathbb{M}_{p}$ can be different from $\mathbb{M}_{q}$ if $p \neq q$. So, in this approximation B is constructed from $K M_{\mathrm{MC}}$ modulated ensemble members. The elements of $\boldsymbol{\gamma}$ must be given by

$$
\boldsymbol{\gamma}_{p}=\left[\frac{1}{K} \sum_{k=1}^{K} \sum_{m \in \mathbb{M}_{k}}\left(\boldsymbol{\chi}_{p}^{(m)}\right)^{2}\right]^{-1 / 2}
$$

to ensure that (4) and (5) are the same if no MC approximation is used (i.e., if $\mathbb{M}_{k}=\mathbb{M}$ for all $k$ ).

\section{Computational cost}

In 4DVAR the matrix B is never explicitly calculated, but always appears in the form of a matrix-vector product. Here we will compare the computational complexity of the matrix-vector computation with the localized background error covariance matrix for three different methods: (i) localization with C constructed using Gaspari and Cohn (1999) fifth-order rational functions followed by a standard matrix-vector multiplication, (ii) localization using Bishop et al. (2011) methodology assuming $\mathbf{C}^{1 / 2}$ is readily available, and (iii) MC localization as described above. As in section 2 we assume that localization is applied in a finite-difference model with a structured grid that has $N_{x}$ points in the horizontal $x$ direction, $N_{y}$ in the horizontal $y$ direction, $N_{z}$ in the vertical $z$ direction, and that $N_{r}$ quantities are defined at each grid point, giving the model a total dimensionality of $N=N_{x} N_{y} N_{z} N_{r}$. In all three cases an arbitrary $N$-dimensional vector used in the calculation of the matrix-vector product is denoted as $\mathbf{v}$. The number of raw ensemble members is $K$. The $O(Z)$ notation is used to indicate that the number of computational operations (addition, multiplication, exponentiation, etc.) is smaller than $c Z$ as $Z \rightarrow \infty$, where $c>0$ is a scalar.

The calculation of the ensemble mean $\overline{\mathbf{x}}$ and the deviations of the ensemble members from the ensemble mean requires $O(K N)$ operations in all considered methods. When using Gaspari and Cohn (1999), it is convenient to rewrite the $N$-dimensional vector $\left(\mathbf{B}_{\text {ens }}{ }^{\circ} \mathbf{C}\right) \mathbf{v}$ as

$$
\frac{1}{K-1} \sum_{k=1}^{K}\left(\mathbf{x}^{(k)}-\overline{\mathbf{x}}\right) \circ\left(\mathbf{C}\left[\left(\mathbf{x}^{(k)}-\overline{\mathbf{x}}\right) \circ \mathbf{v}\right]\right) .
$$

The calculation of each of the two $N$-dimensional Schur products in (7) requires $O(N)$ operations per ensemble member. Calculation of the inner product of the $i$ th row of the sparse $N \times N$ matrix $\mathbf{C}$ with $\left(\mathbf{x}^{(k)}-\overline{\mathbf{x}}\right) \circ \mathbf{v}$ requires $L_{G}$ operations, where $L_{G}$ is the number of grid points that lie in the compact support of the fifth-order rational function. The summation of the $N$-dimensional terms in (7) requires an additional $O(K N)$ operations. In total, we find that the direct implementation of (7) requires

$$
O(K N)+O(K N)+O\left(K N L_{G}\right)+O(K N) \sim O\left(K N L_{G}\right)
$$

operations.

Bishop et al. (2011) describe an efficient method for ensemble covariance localization when $\mathbf{C}^{1 / 2}$ is readily available and each element is the product of one of $M_{h}$ horizontal basis functions with one of $M_{z}$ vertical basis functions. First, the $N$-dimensional vector $\left(\mathbf{x}^{(k)}-\overline{\mathbf{x}}\right) \circ \mathbf{v}$ needs to be calculated at the expense of $O(N)$ operations per ensemble member. Next, the different quantities are summed $[O(N)$ computations per ensemble member] followed by the calculation of the inner product of the resulting $N$-dimensional vector with each vertical basis function $\left[O\left(N_{x} N_{y} N_{z} M_{z}\right)\right.$ operations], producing an $N_{x} N_{y} \times M_{z}$ matrix. Then the inner-product of each column of this matrix with the horizontal basis functions is obtained $\left[O\left(N_{x} N_{y} M_{z} M_{h}\right)\right.$ operations], resulting in a vector of size $M_{h} M_{z}$. Subsequently, the transpose of the aforementioned operations is applied in reverse order. Finally, the $K N$-dimensional vectors obtained in this way are summed together at the expense of $O(K N)$ operations. In total this will require

$$
\begin{aligned}
& O(K N)+2 K\left[O(N)+O(N)+O\left(N_{x} N_{y} N_{z} M_{z}\right)\right. \\
& \left.\quad+O\left(N_{x} N_{y} M_{z} M_{h}\right)\right] \sim O\left[K N \frac{M_{z}}{N_{r}} \max \left(1, \frac{M_{h}}{N_{z}}\right)\right]
\end{aligned}
$$

operations.

For MC localization we first need to calculate the $N$-dimensional vector $\gamma$ at the expense of $O\left(K M_{\mathrm{MC}} L_{\mathrm{MC}}\right)$ operations, where $L_{\mathrm{MC}}$ is the number of grid points in the support of one of the vectors $\boldsymbol{\chi}^{(m)}$. Calculation of the $N$-dimensional vectors $\left(\mathbf{x}^{(k)}-\overline{\mathbf{x}}\right) \circ \boldsymbol{\gamma}$ and $\left(\mathbf{x}^{(k)}-\overline{\mathbf{x}}\right) \circ \boldsymbol{\gamma} \circ \mathbf{v}$ requires an additional $O(N)$ operation for each ensemble member. The computation of $\mathbf{B}_{\mathrm{MC}} \mathbf{v}$ is then completed by selecting $M_{\mathrm{MC}}$ pieces from each of these vectors, with 
each piece containing $L_{\mathrm{MC}} N_{r}$ elements, and adding these parts together. This requires $O\left(K M_{\mathrm{MC}} L_{\mathrm{MC}} N_{r}\right)$ operations. Hence, the total number of operations required is

$$
\begin{aligned}
& O(K N)+O\left(K M_{\mathrm{MC}} L_{\mathrm{MC}}\right)+O(K N)+O\left(K M_{\mathrm{MC}} L_{\mathrm{MC}} N_{r}\right) \\
& \sim O\left[K N \max \left(1, \frac{M_{\mathrm{MC}} L_{\mathrm{MC}}}{N_{x} N_{y} N_{z}}\right)\right] .
\end{aligned}
$$

A comparison of (8) and (10) shows that MC localization is less computationally intensive than Gaspari and Cohn (1999) if $M_{\mathrm{MC}} L_{\mathrm{MC}} / N_{x} N_{y} N_{z} L_{G}<1$. So, if the compact supports are of comparable size in the two methods $\left(L_{\mathrm{MC}} \approx L_{G}\right)$ and if the size of $\mathbb{M}_{k}$ is chosen to be smaller than the number of grid points, MC localization is computationally more efficient than using $\mathbf{C}$ generated with Gaspari and Cohn (1999). Comparison of (9) and (10) shows that MC localization is computationally more efficient than Bishop et al. (2011) if $M_{\mathrm{MC}} L_{\mathrm{MC}} N_{r} / M_{z} M_{h} N_{x} N_{y}<1$. No general statement can be made whether this inequality is indeed satisfied, as $M_{z}, M_{h}, M_{\mathrm{MC}}$, and $L_{\mathrm{MC}}$ will vary depending on $\mathbf{C}^{1 / 2}$ used and the number of modulated ensemble members in the $\mathrm{MC}$ approximation, but choices for which the inequality is satisfied do exist. For example, if the case in which no MC approximation is used $\left(M_{\mathrm{MC}}=N_{x} N_{y} N_{z}\right)$ is compared with a case in which localization takes place in spectral space $\left(M_{h} M_{z}=N_{x} N_{y} N_{z}\right)$ as is done, for example, in Gauthier et al. (1998) and Kuhl et al. (2013) for the horizontal directions, the ratio of the operation counts between MC localization and Bishop et al. (2011) is $L_{\mathrm{MC}} N_{r} / N_{x} N_{y}$, which is smaller than 1 if the horizontal (vertical) localization scale is much smaller than the horizontal (vertical) extent of the model.

\section{MC localization in a $1 \mathrm{D}$ model}

In a strong-constraint 4DVAR system where only the initial conditions are corrected, the data assimilation correction at time $t_{0}$ is found by minimizing the following cost function:

$$
\begin{aligned}
J= & \frac{1}{2} \delta \mathbf{x}\left(t_{0}\right)^{\mathrm{T}} \mathbf{B}^{-1} \delta \mathbf{x}\left(t_{0}\right) \\
& +\frac{1}{2}\left[\mathbf{d}-\mathbf{H M} \delta \mathbf{x}\left(t_{0}\right)\right]^{\mathrm{T}} \mathbf{R}^{-1}\left[\mathbf{d}-\mathbf{H M} \delta \mathbf{x}\left(t_{0}\right)\right]
\end{aligned}
$$

(Bennett 1992; Courtier et al. 1994), where the column vector $\delta \mathbf{x}\left(t_{0}\right)$ is the correction to the modeled ocean state, $\mathbf{B}$ is the localized background error covariance in the initial conditions, $\mathbf{R}$ is the observational error covariance, $\mathbf{d}$ is the innovation vector (difference between observations made during a time window $\left[t_{0}, t_{1}\right]$ and prior model predictions for these observations), $\mathbf{H}$ is the linear operator that samples the model result between $\left[t_{0}, t_{1}\right]$ and generates model predictions for the observations, $\mathbf{M}$ is the tangent linear model operator that takes the model perturbation at $t=t_{0}$ as input and outputs model results for the period $\left[t_{0}, t_{1}\right]$ using a linearized version of the model equations, and $\mathbf{M}^{\mathrm{T}}$ is its adjoint. Repeated minimizations of (11) with updated linearizations $\mathbf{M}$ and initial conditions are necessary if one wants to account for nonlinearities in the model. Here $J$ obtains its minimum for

$$
\delta \mathbf{x}\left(t_{0}\right)=\mathbf{B M}^{\mathrm{T}} \mathbf{H}^{\mathrm{T}}\left(\mathbf{H M B M}^{\mathrm{T}} \mathbf{H}^{\mathrm{T}}+\mathbf{R}\right)^{-1} \mathbf{d}
$$

(Courtier 1997). From (12) it follows that when B is constructed using (4) or (5), the model correction $\delta \mathbf{x}\left(t_{0}\right)$ is a weighted combination of the modulated ensemble members $\left(\mathbf{x}^{(k)}-\overline{\mathbf{x}}\right) \circ \boldsymbol{\chi}^{(m)} \circ \boldsymbol{\gamma}$. In this section we will (i) investigate how well $\mathbf{B}_{\mathrm{MC}}$ approximates $\mathbf{B}_{\text {full }}$ as $M_{\mathrm{MC}}$, the number of elements in the set $\mathbb{M}_{k}$, changes, and (ii) analyze the power spectral density of the modulated ensemble members. All experiments in this section use a $1 \mathrm{D}$ grid with $N=1001$ grid points and one unitless variable to denote the position in space.

We draw the ensemble members $\mathbf{x}^{(k)}$ for this 1D model by first constructing the Fourier transform, then applying an inverse Fourier transform to it giving a function $x^{(k)}$, and finally evaluating this function at the grid points. The Fourier transform for ensemble member $k$ is created as follows:

$$
\begin{aligned}
\mathscr{F} & x^{(k)}(\kappa) \\
= & \sum_{l=-N_{1 / 2}}^{N_{1 / 2}} A_{l}\left(\frac{2 \pi}{N}\right)^{1 / 2}\left(2 \pi \sigma^{2}\right)^{1 / 4} \exp \left(-\frac{1}{4} \sigma^{2} \kappa_{l}^{2}\right) \delta\left(\kappa-\kappa_{l}\right) .
\end{aligned}
$$

Here $\kappa$ is the wavenumber, $N_{1 / 2}=1 / 2(N-1), \kappa_{l}=$ $2 \pi l / N$, and

$$
A_{l}=\left\{\begin{array}{lll}
=A_{\mathrm{Re}}(0) & \text { if } & l=0 \\
=\frac{1}{\sqrt{2}} A_{\mathrm{Re}}(l)+\frac{i}{\sqrt{2}} A_{\mathrm{Im}}(l) & \text { if } & l>0, \\
=A_{-l}^{*} & \text { if } & l<0
\end{array}\right.
$$

where $i^{2}=-1$ and $A_{\operatorname{Re}}(l), A_{\operatorname{Im}}(l)$ are drawn from a Gaussian distribution with zero mean and unit variance. Then $\left\langle\mathscr{F} x^{(k)}(\kappa)\right\rangle=0$, with $\langle\cdot\rangle$ denoting the expectation value, and hence the true model error mean $\left\langle x^{(k)}\right\rangle=0$. Furthermore, by the Wiener-Khinchin theorem (Engelberg $2007)$ the power spectral density $S\left(x^{(k)}\right)$ is related to the covariance function $\rho$ as follows:

$$
\begin{aligned}
S\left(x^{(k)}\right) & =\left\langle\left|\mathscr{F} x^{(k)}\right|^{2}(\kappa)\right\rangle \\
& =\sum_{l=-N_{1 / 2}}^{N_{1 / 2}} \frac{2 \pi}{N}\left(2 \pi \sigma^{2}\right)^{1 / 2} \exp \left(-\frac{1}{2} \sigma^{2} \kappa_{l}^{2}\right) \delta\left(\kappa-\kappa_{l}\right) \\
& =\mathscr{F} \rho(\kappa) .
\end{aligned}
$$


Taking the inverse Fourier transform of the right-hand side of (15) then shows that the true covariance of the ensemble members created this way is

$$
\begin{aligned}
\rho(p, q) & \stackrel{\text { def }}{=}\left\langle\left(\mathbf{x}_{p}^{(k)}-\left\langle\mathbf{x}_{p}^{(k)}\right\rangle\right)\left(\mathbf{x}_{q}^{(k)}-\left\langle\mathbf{x}_{q}^{(k)}\right\rangle\right)\right\rangle \\
& =\sum_{l=-N_{1 / 2}}^{N_{1 / 2}} \frac{2 \pi}{N}\left(2 \pi \sigma^{2}\right)^{1 / 2} \exp \left[-\frac{1}{2} \sigma^{2} \kappa_{l}^{2}\right] \mathscr{F}^{-1}\left[\boldsymbol{\delta}\left(\kappa-\kappa_{l}\right)\right][d(p, q)] \\
& =\sum_{l=-N_{1 / 2}}^{N_{1 / 2}} \frac{2 \pi}{N} \frac{\sigma}{(2 \pi)^{1 / 2}} \exp \left[-\frac{1}{2} \sigma^{2} \kappa_{l}^{2}\right] \exp \left[i \kappa_{l} d(p, q)\right] \\
& \approx \int_{-\infty}^{\infty} \frac{\sigma}{(2 \pi)^{1 / 2}} \exp \left[-\frac{1}{2} \sigma^{2} \kappa_{l}^{2}\right] \exp [i \kappa d(p, q)] d \kappa=\exp \left[-\frac{1}{2} \frac{d(p, q)^{2}}{\sigma^{2}}\right],
\end{aligned}
$$

where $d(p, q)$ is the (dimensionless) distance between the grid points $p$ and $q$ and the integral approximation in the last line of (16) holds if $2 \pi \sigma / N \ll 1$ (necessary to replace the sum with an integral) and $\pi \sigma \gg 1$ (necessary to allow extension of the integral boundaries to $\pm \infty)$.

\section{a. Convergence (dependence on $M_{\mathrm{MC}}$ )}

Decrease in $M_{\mathrm{MC}}$ lowers computation cost, but is expected to increase the sampling error in $\mathbf{B}_{\mathrm{MC}}$. In this subsection we consider the dependence of the relative error $\left\|\mathbf{B}_{m} \mathbf{v}-\mathbf{B}_{\text {full }} \mathbf{v}\right\|\left\|\mathbf{B}_{\text {full }} \mathbf{v}\right\|^{-1}$ on $M_{\mathrm{MC}}$ for typical vectors $\mathbf{v}$. As inversion in (12) is performed using an iterative method (e.g., conjugate gradient), $\mathbf{B}$ is repeatedly applied to vectors of the form $\mathbf{M}^{\mathrm{T}} \mathbf{H}^{\mathrm{T}} \mathbf{r}$ where $\mathbf{r}$ is the residual. In 4DVAR it is assumed that $\mathbf{r}$ follows a Gaussian distribution with covariance $\mathbf{H M B M}^{\mathrm{T}} \mathbf{H}^{\mathrm{T}}+\mathbf{R}$ and zero mean (Bennett 1992) and hence under these assumptions $\mathbf{M}^{\mathrm{T}} \mathbf{H}^{\mathrm{T}} \mathbf{r}$ itself follows a Gaussian distribution with covariance $\mathbf{M}^{\mathrm{T}} \mathbf{H}^{\mathrm{T}}\left(\mathbf{H} \mathbf{M B} \mathbf{M}^{\mathrm{T}} \mathbf{H}^{\mathrm{T}}+\mathbf{R}\right) \mathbf{H M}$ and zero mean. In this paper we will neglect the implementationdependent intricacies contained in $\mathbf{M}, \mathbf{H}$, and $\mathbf{R}$ and simply construct the typical vectors $\mathbf{v}$ by drawing them from a Gaussian distribution with zero mean and with the identity matrix as covariance.

The relative error introduced by the Monte Carlo approximation has been calculated for different values of $M_{\mathrm{MC}}$. In our base experiment $L_{\mathrm{MC}}=101$, where $L_{\mathrm{MC}}$ is the maximum number of grid points in the support of the $N$-dimensional vectors $\boldsymbol{\chi}^{(m)}$. We draw a 10 -member ensemble $(K=10)$ with covariance (16), an $N$-dimensional vector $\mathbf{v}$ from a standard normal distribution, and calculate the relative error $\left\|\mathbf{B}_{m} \mathbf{v}-\mathbf{B}_{\text {full }} \mathbf{v}\right\|\left\|\mathbf{B}_{\text {full }} \mathbf{v}\right\|^{-1}$. This procedure is repeated 1000 times. The mean of the relative error is shown in Fig. 2 as a solid black line. A nonlinear least squares fit to the average relative error finds that it scales as $\sim M_{\mathrm{MC}}^{-0.51}$, close to the $\sim M_{\mathrm{MC}}^{-1 / 2}$ expected for a Monte Carlo approximation (Geyer 1992).
The same experiment has been repeated using $K=5$ and $K=20$ and the results are also shown in Fig. 2. Nonlinear fits to these lines show that they have similar $\sim M_{\mathrm{MC}}^{-0.5}$ dependence on $M_{\mathrm{MC}}$, but that for $K=5$ the relative error is $25 \% \pm 2 \%$ larger than for the case $K=10$, while for the case $K=20$ the relative error is $26 \% \pm 1 \%$ smaller. The relation between the mean relative error on one hand and $K$ and $M_{\mathrm{MC}}$ on the other hand is further explored in Fig. 3. For $\left(K, M_{\mathrm{MC}}\right) \in\{5,10, \ldots, 50\} \times$ $\{10,20, \ldots, 100,150, \ldots, 500,600, \ldots, 1000\}$ the relative error $\left\|\mathbf{B}_{m} \mathbf{v}-\mathbf{B}_{\text {full }} \mathbf{v}\right\|\left\|\mathbf{B}_{\text {full }} \mathbf{v}\right\|^{-1}$ is calculated for 1000 realizations and then averaged. Each of these averages is represented by one of the $10 \times 23=230$ dots in Fig. 3. This figure shows that the relative error scales with $\sim M_{\mathrm{MC}}^{-0.5}$ if $K$ is kept fixed. However, it also shows that the mean relative error can be bounded by a function proportional to $\left(K M_{\mathrm{MC}}\right)^{-0.5}$. As $K$ becomes smaller the mean relative error attains values below the upper bound. This can be explained by the fact that modulated ensemble members created from the same raw ensemble member are better approximations of each other than modulated ensemble members created from different raw ensemble members. For example, vectors $\left(\mathbf{x}^{(k)}-\overline{\mathbf{x}}\right) \circ \boldsymbol{\chi}^{(m)} \circ \boldsymbol{\gamma}$ and $\left(\mathbf{x}^{(k)}-\overline{\mathbf{x}}\right) \circ \boldsymbol{\chi}^{(m+1)} \circ \boldsymbol{\gamma}$, where $m$ and $m+1$ are neighboring points, are different in at most two elements while $\left(\mathbf{x}^{(k)}-\overline{\mathbf{x}}\right) \circ \boldsymbol{\chi}^{(m)} \circ \boldsymbol{\gamma}$ and $\left(\mathbf{x}^{(k+1)}-\overline{\mathbf{x}}\right) \circ \boldsymbol{\chi}^{(m)} \circ \boldsymbol{\gamma}$ can be different in as many as $L_{\mathrm{MC}}$ elements. In all cases in this experiment a mean relative error of less than 0.1 is reached if $K M_{\mathrm{MC}} \geq 3500$.

So far the elements of the vector $\mathbf{v}$ were drawn from a normal distribution. If observations only cover part of the domain, $\mathbf{H}$ will be sparse and it is possible that the vector $\mathbf{v} \approx \mathbf{M}^{\mathrm{T}} \mathbf{H}^{\mathrm{T}} \mathbf{r}$ mainly consists of zeros. To test the effect of such a structure of $\mathbf{v}$ on aforementioned results, the previous experiment has been repeated using a 10 -member ensemble $(K=10)$ with $\mathbf{v}$ obtained by drawing from the distribution in (16) and then randomly setting $90 \%$ of its elements to zero. The 


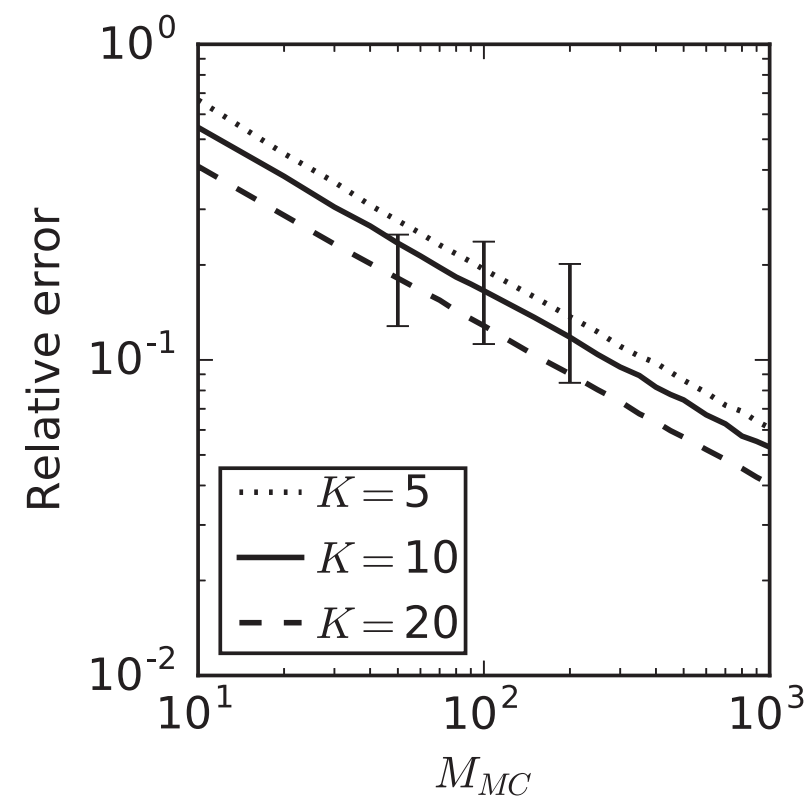

FIG. 2. The relative error $\left\|\mathbf{B}_{\text {full }} \mathbf{v}-\mathbf{B}_{\mathrm{MC}} \mathbf{v}\right\|\left\|\mathbf{B}_{\text {full }} \mathbf{v}\right\|^{-1}$ as function of $M_{\mathrm{MC}}$ (the size of the sets $\mathbb{M}_{k}$ in the MC approximation) and different choices of $K$ (the number of raw ensemble members); note that $K M_{\mathrm{MC}}$ is the total number of modulated ensemble members used. The solid line shows the mean relative error averaged over 1000 different realizations of the raw ensemble, the modulated ensemble, and the vector $\mathbf{v}$. Error bars indicate the interval around the mean in which $90 \%$ of the relative errors fall.

mean relative error still scales with $\sim M_{\mathrm{MC}}^{-0.5}$, but was $0.90 \pm 0.02$ times the mean relative error found in the previous experiment. So, in this $1 \mathrm{D}$ experiment MC localization does not behave differently when $\mathbf{r}$ is sparse.

\section{b. Localization distance $\left(L_{\mathrm{MC}}\right)$}

The goal of this subsection is to find $L_{\mathrm{MC}}$, the number of grid points in the support of $\boldsymbol{\chi}^{(m)}$, for which the modulated ensemble covariance best approximates the true covariance. For this purpose we have drawn a 20 -member ensemble $(K=20)$ with covariance in (16), used MC localization with $M_{\mathrm{MC}}=150$, thereby creating an ensemble of 3000 modulated members, and calculated the covariance between the point in the center of the domain and its neighboring points. This experiment has been repeated 50 times for $L_{\mathrm{MC}}=61,101,141$. For each choice of $L_{\mathrm{MC}}$, the mean value and standard deviation of these 50 trials are shown in Fig. 4 together with the true covariance and the nonlocalized ensemble covariance. The nonlocalized ensemble covariance (Fig. 4a) does not tend to zero at large distances, but exhibits spurious long distance covariances with a typical size of 0.2 (dashed lines). The MC localization is successful at removing these spurious covariances. The

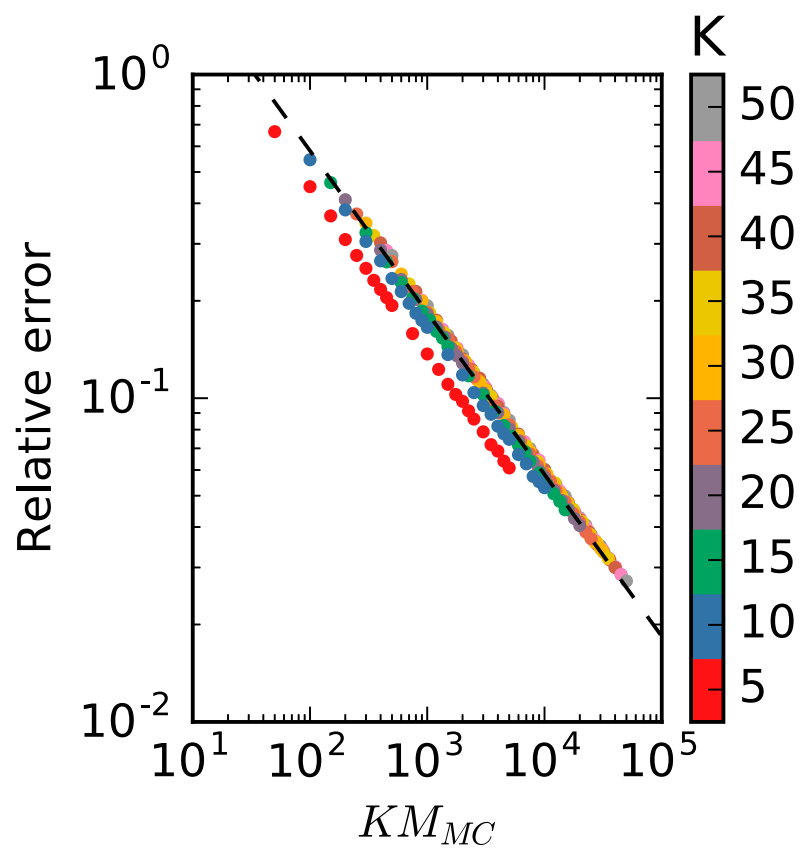

FIG. 3. Each dot represents the mean relative error $\left\|\mathbf{B}_{\text {full }} \mathbf{v}-\mathbf{B}_{\mathrm{MC}} \mathbf{v}\right\|\left\|\mathbf{B}_{\text {full }} \mathbf{v}\right\|^{-1}$ over 1000 realizations of $\mathbf{B}_{\text {full }}, \mathbf{B}_{\mathrm{MC}}$, and $\mathbf{v}$ for a different value of $K$ and $M_{\mathrm{MC}}$. Colors show the size of the raw ensemble $K$. The dashed line follows the relation 5.8(KMC $)^{-1 / 2}$.

figure also shows that on average the width of the localized covariances is smaller than that of the true covariance. This narrowing is most pronounced for $L_{\mathrm{MC}}=61$. It is less for $L_{\mathrm{MC}}=141$, but the larger standard deviations in the tails show that some of the modulated ensembles generated during the 50 trials have a nonzero covariance where the true covariance is nearly zero. This suggests that there is some optimal value for $L_{\mathrm{MC}}$, hereafter referred to as $\hat{L}_{\mathrm{MC}}$, for which the root-mean-square (RMS) error between the true covariance as given in (16) and the localized ensemble covariance is minimal. Repeating this experiment using different values of $\sigma$ and $L_{\mathrm{MC}}$ with $K M_{\mathrm{MC}}=4000$ shows that this is indeed the case. The found values for $\hat{L}_{\mathrm{MC}}$ are shown in Fig. 5. A linear fit to the data shows that $\hat{L}_{\mathrm{MC}}=(3.6 \pm 0.2) \sigma$. In a similar experiment with fixed $K M_{\mathrm{MC}}=4000$, but with different $K$ similar linear relations are found, but with different proportionality constants. As Table 1 shows, the proportionality constant remains between 3 and 4 for a small ensemble $(K \leq 30)$, but starts increasing when the number of raw ensemble members increases. This is in agreement with the increase in optimal localization scales observed in Hamill et al. (2001) and Kirchgessner et al. (2014). An experiment with $K=20$ and $M_{\mathrm{MC}}$ varying between 100 and 400 gives proportionality coefficients between $3.4 \pm 0.2$ and $4.1 \pm 0.2$ with no clear dependence on $M_{\mathrm{MC}}$. 

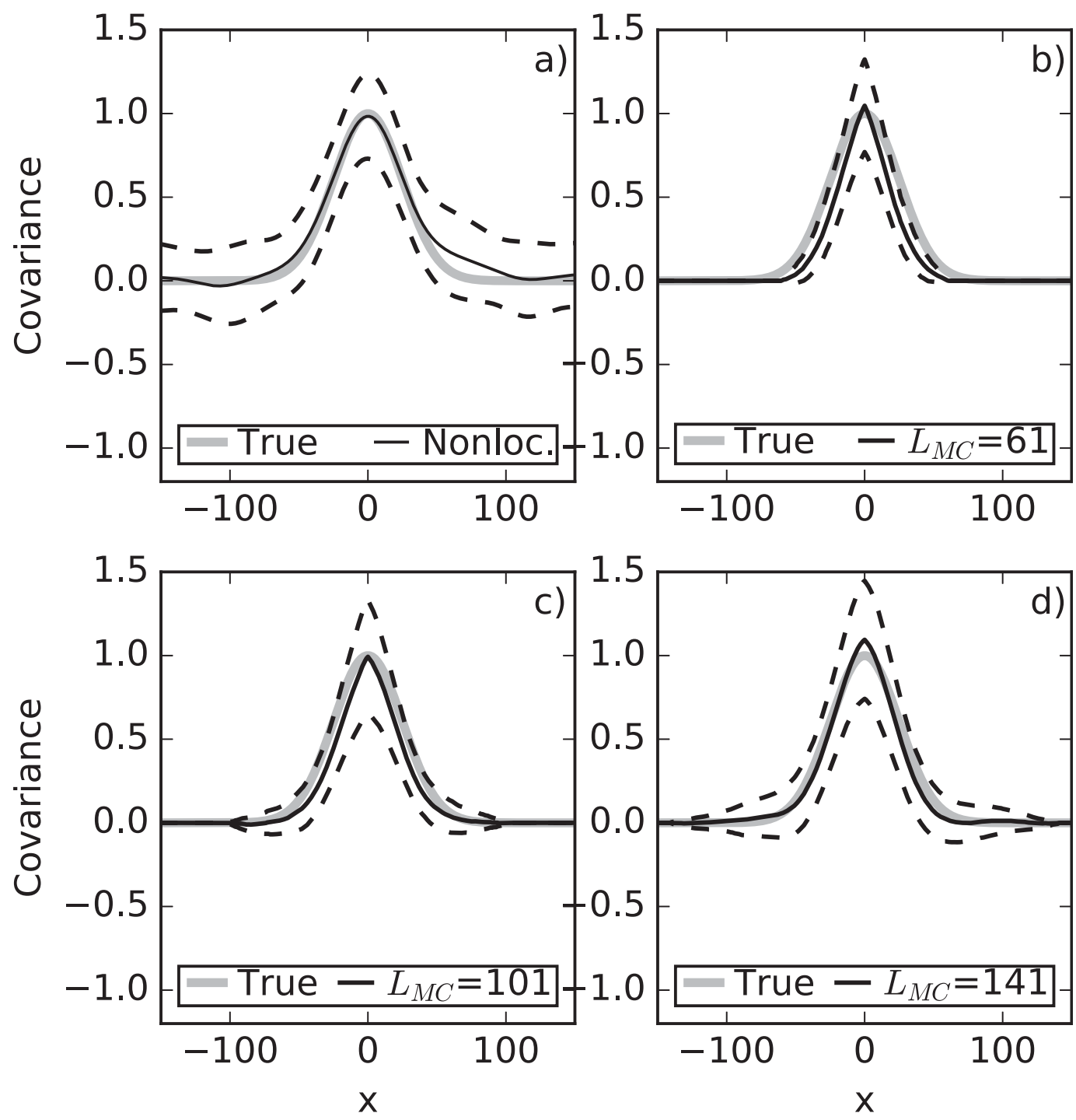

FIG. 4. The comparison of the true (gray) and ensemble-generated covariances (black) computed with respect to the domain center point. (a) Mean (solid black line) \pm standard deviation (dashed black lines) of 50 raw ensemble covariances. (b) Mean (solid black line) \pm standard deviation (dashed black lines) of 50 modulated ensemble covariances with compact support length $L_{\mathrm{MC}}=61$. (c) As in (b), but now $L_{\mathrm{MC}}=101$. (d) As in (b), but with $L_{\mathrm{MC}}=141$.

\section{c. Shape of covariance}

The covariance function in (16) is positive. In reality a negative background error covariance might be expected. For example, when the strength of the circulation around an eddy varies in different ensemble members, the background errors in a component of the horizontal velocity field on different sides of the eddy will be negatively correlated with each other. To test the effect of MC localization in this case, the foregoing experiment is repeated, but now with raw ensemble members constructed using

$$
\mathscr{F} x^{(k)}(\kappa)=\sum_{l=-N_{1 / 2}}^{N_{1 / 2}}\left\{A_{l}\left(\frac{2 \pi}{N}\right)^{1 / 2}\left(2 \pi \sigma^{2}\right)^{1 / 4} \sqrt{\frac{1}{2} \exp \left[-\frac{1}{2}\left(\sigma \kappa_{l}-\pi\right)^{2}\right]+\frac{1}{2} \exp \left[-\frac{1}{2}\left(\sigma \kappa_{l}+\pi\right)^{2}\right]} \delta\left(\kappa-\kappa_{l}\right)\right\}
$$




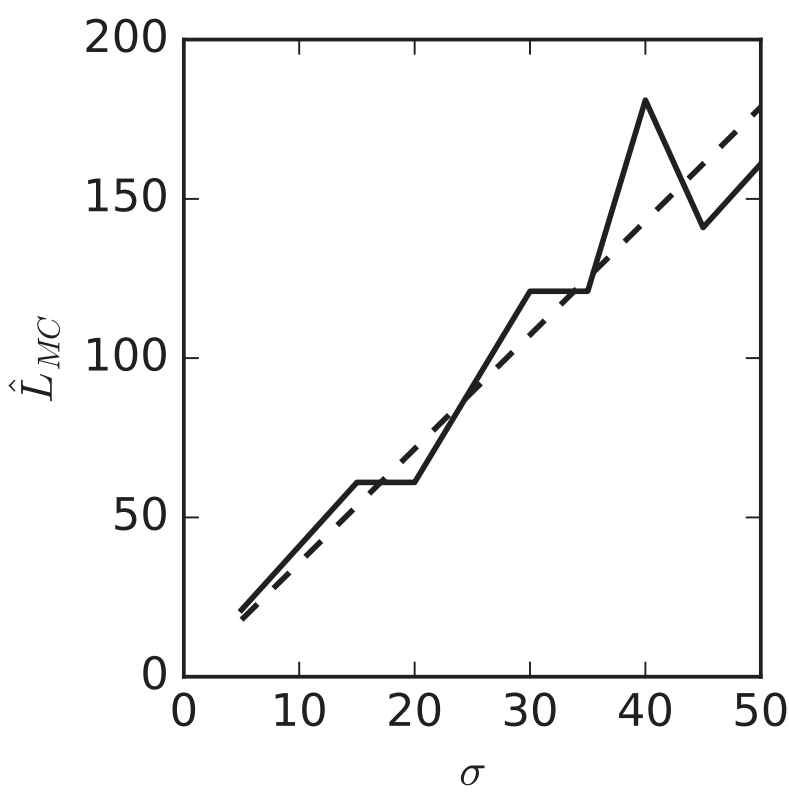

FIG. 5. The relation between $\sigma$ as appearing in (16) and the value for $L_{\mathrm{MC}}$ for which the RMS error between the true covariance and the localized ensemble covariance is minimal. The dashed line shows the linear fit to the data.

with $A_{l}$ as defined in (14). Following a similar procedure as outlined for (15) and (16) this creates raw ensemble members with a covariance function given by

$$
\rho[d(p, q)] \approx \cos \left[\frac{d(p, q) \pi}{\sigma}\right] \exp \left[-\frac{d(p, q)^{2}}{2 \sigma^{2}}\right] .
$$

Figure 6 shows that with a localization width $L_{\mathrm{MC}}=101$ the mean of the localized covariances follows the true covariance. However, the negative covariances are not as strong as in the true covariance or in the nonlocalized sample covariance. As the distance between grid point $p$ and grid point $q$ becomes larger there will be fewer modulated ensemble members that have both points lying in their support. Hence, as $d(p, q)$ becomes larger there will be fewer and fewer terms in (4) for which the $(p, q)$ th element is nonzero. Consequently $\left(\mathbf{B}_{\mathrm{MC}}\right)_{p q}$ will go to zero as well. Although on average the localized covariances are positive for $x= \pm 50$, just as the true covariance, Fig. 6 shows that a fraction of localized covariances constructed in this experiment had the wrong sign at this $x$. This is, however, not unique to localization, as the same happens for a fraction of the nonlocalized ensemble covariances.

\section{d. Spectrum}

When $\mathbf{B}_{\text {full }}$ is calculated, $\boldsymbol{\gamma}^{\circ} \boldsymbol{\chi}^{(m)}$ is a boxcar function when grid point $m$ lies farther than $L_{\mathrm{MC}}$ from the domain boundary. As is shown in the appendix, the amplitude of its Fourier transform then scales with a sinc function with a main lobe half-width proportional to $L_{\mathrm{MC}}^{-1}$. The Fourier transform of the modulated ensemble member is a convolution of this function and the Fourier transform of the raw ensemble member. Consequently, the Fourier transform of the modulated ensemble member is a smoothened version of that of the raw ensemble member.

When the support of $\boldsymbol{\chi}^{(m)}$ contains part of the boundary of the domain or when elements $\gamma$ vary because of the MC approximation, see (6), obtaining the spectrum is not as straightforward. To study the spectrum for this case, 10-member ensembles were drawn and localized using MC localization with $L_{\mathrm{MC}}=101$ and $M_{\mathrm{MC}}=400$. Using the MATLAB periodogram routine, the one-sided power spectrum of the nonlocalized and modulated ensemble members was obtained. These steps were repeated 50 times creating 500 nonlocalized and 500 localized spectra.

The ensemble variance for element $p$ is given by $1 /(K-1) \sum_{k=1}^{K} \sum_{m \in \mathbb{M}_{k}}\left(\mathbf{x}^{(k)}-\overline{\mathbf{x}}\right)_{p}^{2} \boldsymbol{\gamma}_{p}^{2}\left(\boldsymbol{\chi}_{p}^{(m)}\right)^{2}$, where $\boldsymbol{\gamma}$ in (2) and (6) was chosen such that the variance in the localized ensemble for element $p$ is exactly (in the case of $\mathbf{B}_{\text {full }}$ ) or approximately (in the case of $\mathbf{B}_{\mathrm{MC}}$ ) equal to the variance of the raw ensemble. For the raw ensemble $K$ ensemble members contribute to the sum that gives the ensemble variance for element $p$, while for the modulated ensemble $\sum_{k=1}^{K} \sum_{m \in \mathbb{M}_{k}} \boldsymbol{x}_{p}^{(m)}=\boldsymbol{\gamma}_{p}^{-2} K$ members contribute. As usually $\boldsymbol{\gamma}_{p}^{-2}>1$ this means that each modulated ensemble members contributes less to the variance than each raw ensemble member. As the total spectral power is equal to the variance (Oppenheim and Schafer 1989) this implies that the power spectral density of the modulated ensemble members has to be smaller than that of the raw ensemble members. To facilitate the comparison of the power spectra on the same scale, the aforementioned 500 localized power spectra have been rescaled by multiplying them with the average ratio of number of modulated members that contribute to the variance over the number raw ensemble members that contribute (i.e., by $1 / N \sum_{p=1}^{N} \boldsymbol{\gamma}_{p}^{-2}$ ).

The (scaled) mean power spectral density from the 500 selected members as well the $5 \%$ and $95 \%$ percentiles are

TABLE 1 . Proportionality constant $\alpha$ ( \pm standard deviation) between $\hat{L}_{\mathrm{MC}}$ and $\sigma$ as function of the number of raw ensemble members $K$.

\begin{tabular}{cccccccc}
\hline \hline$K$ & 10 & 15 & 20 & 25 & 30 & 40 & 50 \\
\hline$\alpha$ & $3.2 \pm 0.2$ & $3.2 \pm 0.14$ & $3.6 \pm 0.2$ & $4.1 \pm 0.3$ & $3.7 \pm 0.2$ & $4.9 \pm 0.3$ & $4.4 \pm 0.2$ \\
\hline
\end{tabular}



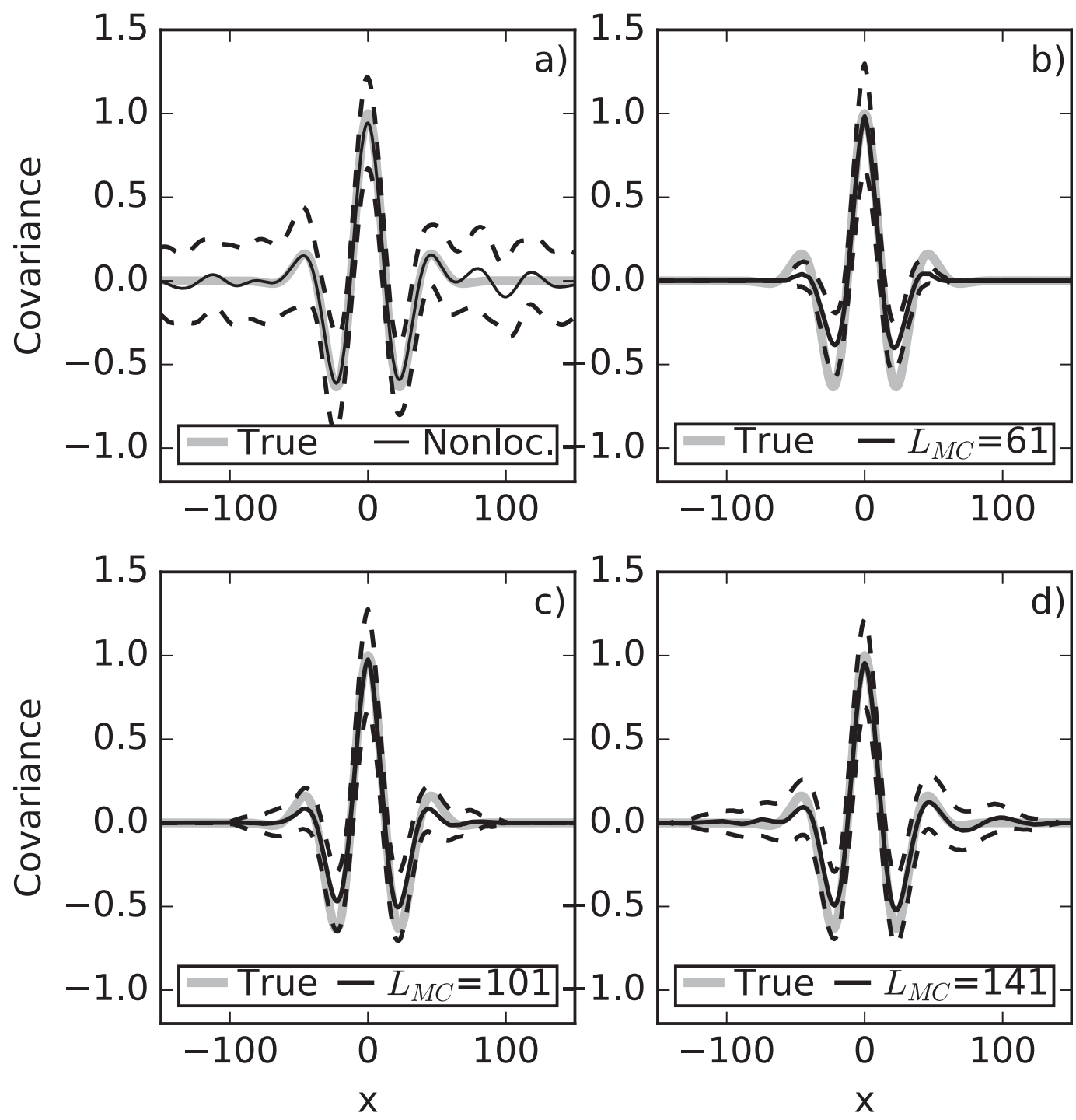

FIG. 6. As in Fig. 4, but now using ensemble members having (18) as true covariance function.

shown in Fig. 7 together with the true spectrum from (15) as a function of the wavenumber $\kappa$. Comparing the averages in the figure shows that the localization scheme suppresses the power spectral density for wavenumbers smaller than $\kappa<0.14$ (i.e., wavenumbers corresponding to wave lengths longer than 45 , or $1 / 2 L_{\mathrm{MC}}$ in our case, by at most $93 \%$ ).

\section{Example from a 3D model}

The experiments in section 4 were all performed on a $1 \mathrm{D}$ grid. We have implemented MC localization in our 3D coastal ocean forecasting model described in section 1 using an ensemble size of $K=50$ and $K M_{\mathrm{MC}}=$ 750000 modulated ensemble members. We use the first baroclinic Rossby radius of deformation, which is $\sim 20 \mathrm{~km}$ for the model region (Chelton et al. 1998), as an estimate for $\sigma$. This, in combination with values for $\alpha$ for large ensembles as found in Table 1 has let us to set $L_{\mathrm{MC}}=100 \mathrm{~km}$ in our model. Figure 8 shows an example of the surface temperature-salinity background error covariance in this model. As perturbations in the flow field alter the advection of river water and as the river outflow is generally fresher and warmer than the ocean this temperature covariance is expected to be negative in and near the river plume. The figure shows that without localization the covariances with the largest magnitude can be found in the areas marked as 1,2 , and 3 . For both the shown and an independently generated 50-member ensemble, the $p$ values of the temperature-salinity correlations have been calculated using MATLAB's corrcoef routine. The area within the dashed lines shows where correlations were significantly 


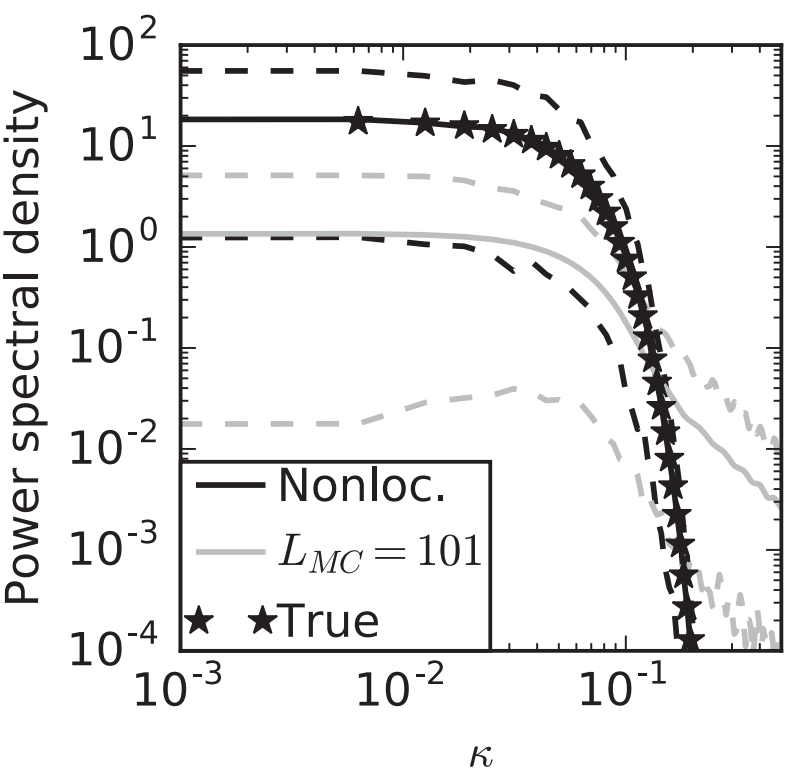

FIG. 7. Power spectral density as a function of the wavenumber $\kappa$ for raw ensemble members (black) and for the modulated ensemble members with $L_{\mathrm{MC}}=101$ and $M_{\mathrm{MC}}=400$ (gray). Solid lines show the power spectral density averaged over 500 ensemble members. Dashed lines show the 5\% and $95 \%$ percentiles. The power spectrum of raw ensemble members as given by (15) is shown as a line of stars.

different from zero at the $90 \%$-confidence level in both ensembles. Apart from a small patch, the covariances in areas 2 and 3 are nonsignificant and MC localization removes them successfully, while it maintains the nonzero, negative covariances in area 1 that are significant.

\section{Summary and conclusions}

Ensemble estimates of the background model error covariance contain spurious long-distance correlations that can degrade the performance of data assimilation algorithms. For our 4DVAR data assimilation system we sought a method to remove these correlations and that is computationally more efficient than popular localization methods like taking the Schur product of the ensemble sample covariance with C generated by Gaspari and Cohn (1999) or the algorithm outlined by Bishop and Hodyss (2011). With this aim we introduced MC localization, which rewrites the localized background error covariance as the sample covariance of a modulated (truncated) ensemble, in which the modulated ensemble members are created by selecting parts of size $L$ from raw ensemble members. The order estimates of the computational cost show that MC localization is computationally more efficient than using $\mathbf{C}$ generated by Gaspari and Cohn (1999). Whether MC localization is computationally more efficient than using Bishop and Hodyss (2011) depends on the details of the implementation used. In particular, computationally efficient MC localization requires that the number of modulated ensemble members that is used in the calculation of the localized background covariance is small. From a test on a $1 \mathrm{D}$ grid with 1000 grid points and a background error covariance shaped as a Gaussian it was found that for this case a MC approximation of the localized background covariance can be created using only a limited number of modulated ensemble members. For the case studied we found that the mean relative error introduced by this MC approximation has an upper bound that scales as $\sim\left(K M_{\mathrm{MC}}\right)^{-1 / 2}$, where $K$ is the number of raw ensemble members and $M_{\mathrm{MC}}$ is the number of modulated ensemble members created out of each raw ensemble member. Using this approximation MC localization was successful in removing spurious long-distance covariances in the 1D model as well as in the 3D example.

For our 1D case, we found that the optimal localization width $L_{\mathrm{MC}}$ increases with increasing length scale of the background errors. This relation qualitatively agrees with the findings by Houtekamer and Mitchell (2001). They calculated RMS errors for their ensemble Kalman filter data assimilation system in which localization is performed by taking the Schur product with $\mathbf{C}$ generated by Gaspari and Cohn (1999) and found that the localization radius for which this error is minimal increases with increasing number of ensemble members. They attributed this to larger distance over which covariances can be computed accurately (Houtekamer and Mitchell 1998). The more accurate computation is possible because the small correlations found between points spaced far apart become distinguishable as the statistical noise level drops with increasing number of ensemble members.

The rescaled power spectrum of the modulated ensemble members was found to be a smoothened version of the spectrum of the nonlocalized ensemble members. In particular, the rescaled power spectrum for the modulated ensemble members contains relatively more power for large wavenumbers, while for small wavenumbers spectral power is strongly reduced compared to the nonlocalized spectrum. This smoothing was shown to be due to the fact that the Fourier transform of most modulated ensemble members is a convolution of the Fourier transform of the nonlocalized ensemble member with a function that scales with the sinc function. In this sense the effect of localization on the spectrum of the ensemble members is found to be comparable to that of windowing. This suggests that in future research it might be interesting to study the effects of varying the strength of the smoothing by using functions other than the boxcar for $\boldsymbol{\chi}^{(m)}$. 

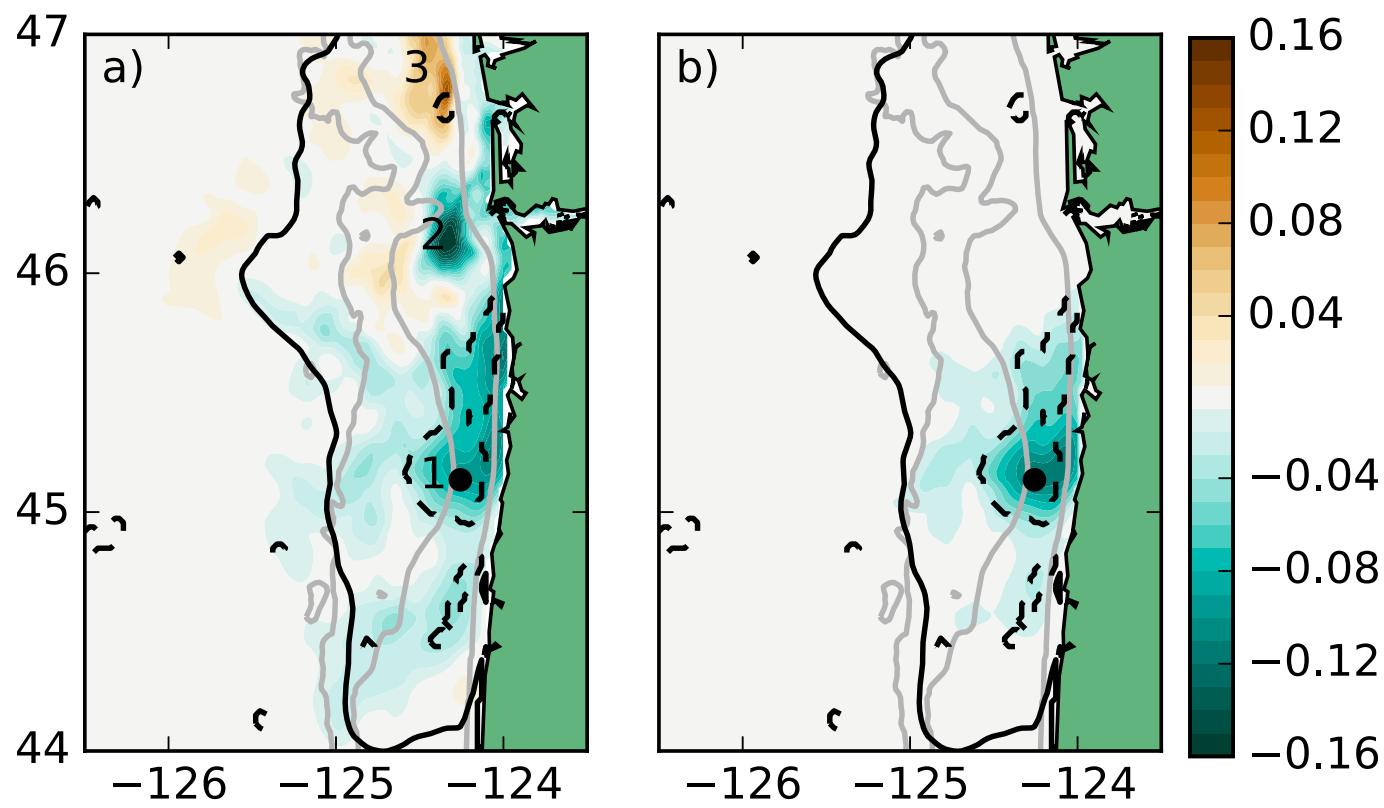

FIG. 8. Ensemble covariance (in $10^{-3}{ }^{\circ} \mathrm{C}$ ) at 0000 UTC 4 May 2011 between the sea surface temperature at the location marked by the dot and the sea surface salinity field in the Columbia River plume (black line is $31.5 \mathrm{psu}$ ). (a) For a nonlocalized 50-member ensemble. (b) For the same 50-member ensemble but now using MC localization with $M_{\mathrm{MC}}=15000$ and $L_{\mathrm{MC}}=51$ in both zonal and meridional direction corresponding to a localization distance of $100 \mathrm{~km}$. In the area enclosed by the dashed lines the nonlocalized sample correlation is significantly different from zero at the $90 \%$ level. The $200-, 1000-$, and $2000-\mathrm{m}$ isobaths are shown as gray lines.

A well-known drawback of the use of localization schemes in data assimilation is their tendency to generate corrections in which the flow is not in geostrophic balance (Mitchell et al. 2002; Lorenc 2003). Though not addressed in this paper, MC localization is expected to produce unbalanced corrections: even if the ensemble perturbations $\mathbf{x}^{(k)}-\overline{\mathbf{x}}$ are in geostrophic balance, a modulated ensemble member will contain a discontinuity in pressure near the boundary of the boxcar function with no geostrophic velocity to match it. Ways to mitigate the imbalance, like using smoother functions for $\boldsymbol{\chi}^{(m)}$ should be addressed in future work.

Another possible topic for future research is the use of multiple localization scales in MC localization. In this paper $\boldsymbol{\chi}^{(m)}$ was assumed to be independent of index $m$ marking the location of the support (i.e., the localization distance was assumed to be homogeneous in space). Several inhomogeneous localization schemes have been developed in which $\mathbf{C}$ is derived from the ensemble (Bishop and Hodyss 2007, 2009a, 2011). It was found that at least in simple systems in which the scale of the error changes in space and in time these schemes outperform nonadaptive localization. Contrary to Bishop and Hodyss (2007) and Bishop and Hodyss (2009a), MC localization does not provide a way to estimate the correlation length scale. However, when local estimates of correlation scales are available, inhomogeneity is expected to be easily implementable in MC localization by making $L_{\mathrm{MC}}$ a function of $m$.

Acknowledgments. This work was supported by the NOAA Coastal Ocean Modeling Testbed (COMT) Grant NA13NOS0120139, Integrated Ocean Observing System/ Northwest Association of Networked Ocean Observing Systems (IOOS/NANOOS) Grant NA16NOS0120019, and the NASA SWOT Science Definition Team Project Grant NNX13AD89G. It used the Extreme Science and Engineering Discovery Environment (XSEDE) TACC Stampede supercomputer, which is supported by National Science Foundation Grant ACI-1053575, through allocation OCE160001. The authors also would like to thank R. Miller, A. Moore, and two anonymous reviewers for their comments on the manuscript.

\section{APPENDIX}

\section{Fourier Transform of the Modulated Ensemble Members}

As in section 4 consider a uniform grid with spatial coordinate $\xi$ and grid spacing $d \xi$. To simplify the derivation of the Fourier transform of the modulated ensemble member $\mathbf{x}^{(k)} \circ \boldsymbol{\chi}^{(m)} \circ \boldsymbol{\gamma}$ we extend $\boldsymbol{\chi}^{(m)}, \boldsymbol{\gamma}$ to all $\xi \in \mathbb{R}$ by defining functions 
$\chi^{(m)}(\xi), \quad \gamma(\xi)$ with $\chi^{(m)}(\xi)=1$ if $\left|\xi-\xi_{m}\right| \leq 1 / 2 L_{\mathrm{MC}}$, with \lfloor\rfloor as the floor function. Hence, $\gamma(\xi)=$ $\gamma(\xi)=\left[1 / K \sum_{k=1}^{K} \sum_{m \in \mathbb{M}} \chi^{(m)}(\xi)^{2}\right]^{-1 / 2}=\left[\sum_{m=1}^{M} \chi^{(m)}(\xi)^{2}\right]^{-1 / 2} . \quad\left(\left\lfloor L_{\mathrm{MC}} / d \xi\right\rfloor+1\right)^{-1 / 2} \stackrel{\text { def }}{=} c_{\gamma}$ for such $\xi$. If $m$ is such $\gamma(\xi)=\left[1 / K \sum_{k=1} \sum_{m \in \mathbb{M}} \chi^{(m)}(\xi)\right]=\left[\sum_{m=1} \chi^{(m)}(\xi)\right] \cdot$ that $\left|\xi_{m}-\xi_{1}\right|,\left|\xi_{m}-\xi_{N}\right|>L_{\mathrm{MC}} \quad \chi^{(m)}(\xi)=0 \quad$ if Then $\chi^{(m)}\left(\xi_{p}\right)=\boldsymbol{\chi}_{p}^{(m)}$ and $\gamma\left(\xi_{p}\right)=\boldsymbol{\gamma}_{p}$.

If $\left|\xi-\xi_{1}\right|,\left|\xi-\xi_{N}\right| \geq 1 / 2 L_{\mathrm{MC}}$ then there are $\left|\xi-\xi_{1}\right|,\left|\xi-\xi_{N}\right|<1 / 2 L_{\mathrm{MC}}$ and hence $\chi^{(m)}(\xi) \gamma(\xi)=$ $\left\lfloor L_{\mathrm{MC}} / d \xi\right\rfloor+1$ points for which $\left|\xi-\xi_{p}\right|<1 / 2 L_{\mathrm{MC}}$ $c_{\gamma} \chi^{(m)}(\xi)$ for such $m$. In this case the Fourier transform of $\chi^{(m)} \circ \gamma$ is given by

$$
\begin{aligned}
\mathscr{T}\left(\chi^{(m)} \circ \gamma\right)(\kappa) & =\int_{\mathbb{R}} c_{\gamma} \chi^{(m)}(\xi) \exp (-i \kappa \xi) d \xi=c_{\gamma} \int_{\xi_{m}-1 / 2 L_{\mathrm{MC}}}^{\xi_{m}+1 / 2 L_{\mathrm{MC}}} \exp (-i \kappa \xi) d \xi \\
& =\left.c_{\gamma} \exp \left(-i \kappa \xi_{m}\right) \frac{\exp (-i \kappa \xi)}{-i \kappa}\right|_{\xi=-1 / 2 L_{\mathrm{MC}}} ^{1 / 2 L_{\mathrm{MC}}}=c_{\gamma} L_{\mathrm{MC}} \exp \left(-i \kappa \xi_{m}\right) \operatorname{sinc}\left(\frac{1}{2} L_{\mathrm{MC}} \kappa\right) .
\end{aligned}
$$

Provided the integrals exist the Fourier transform of the modulated ensemble member $x^{(k)}(\xi)$ then follows from the convolution theorem:

$$
\begin{aligned}
& \mathscr{F}\left(x^{(k)} \circ \chi^{(m)} \circ \gamma\right)(\kappa)=\int_{\mathbb{R}} x^{(k)}(\xi)\left(\chi^{(m)} \circ \gamma\right)(\xi) \exp (-i \kappa \xi) d \xi \\
& =\int_{\mathbb{R}} x^{(k)}(\xi)\left[\frac{1}{2 \pi} \int_{\mathbb{R}} \mathscr{F}\left(\chi^{(m)} \circ \gamma\right)\left(\kappa^{\prime}\right) \exp \left(i \kappa^{\prime} \xi\right) d \kappa^{\prime}\right] \exp (-i \kappa \xi) d \xi \\
& =\frac{1}{2 \pi} \iint_{\mathbb{R}^{2}} x^{(k)}(\xi) \exp \left[-i\left(\kappa-\kappa^{\prime}\right) \xi\right] \mathscr{F}\left(\chi^{(m)} \circ \gamma\right)\left(\kappa^{\prime}\right) d \xi d \kappa^{\prime} \\
& =\frac{1}{2 \pi} \int_{\mathbb{R}} \mathscr{F} x^{(k)}\left(\kappa-\kappa^{\prime}\right) \mathscr{F}\left(\chi^{(m)} \circ \gamma\right)\left(\kappa^{\prime}\right) d \kappa^{\prime}=\frac{1}{2 \pi} \mathscr{F} x^{(k) * \mathscr{F}}\left(\chi^{(m)} \circ \gamma\right)(\kappa) \text {. }
\end{aligned}
$$

Here the last line of (A2) shows that the Fourier transform of the modulated ensemble member $x^{(k)} \circ \chi^{(m)} \circ \gamma$ at the wavenumber $\kappa$ is a weighted combination of values of the Fourier transform at nearby wavenumbers $\kappa-\kappa^{\prime}$ where the amplitude of the nearby Fourier component is scaled with $\operatorname{sinc}\left(1 / 2 L_{\mathrm{MC}} \kappa^{\prime}\right)$ and the phase is shifted by $\kappa^{\prime} \xi_{m}$. Notice that for $x^{(k)}$ constructed using (13) or (17), $\mathscr{F} x^{(k)}$ consists of a finite number of delta functions and hence the integrals in (A2) will be properly defined. In (A2) and (A5) the asterisk indicates convolution.

On a discrete grid the value of the modulated ensemble member is only sampled on the grid points and hence the discrete modulated ensemble member can be written as $x^{(k)} \circ \chi^{(m)} \circ \gamma \circ \operatorname{III}(\xi)$ with

$$
\mathrm{III}_{d \xi}(\xi)=\sum_{l=-\infty}^{\infty} \delta(\xi-l d \xi)
$$

with the Dirac Comb or sampling function, which has Fourier transform (Strichartz 1994)

$$
\mathscr{F} \mathrm{III}_{d \xi}(\kappa)=\frac{2 \pi}{d \xi} \mathrm{III}_{\frac{2 \pi}{d \xi}}(\kappa)=\frac{2 \pi}{d \xi} \sum_{l=-\infty}^{\infty} \delta\left(\kappa-l \kappa_{\text {sample }}\right) .
$$

Here $\kappa_{\text {sample }}=2 \pi / d \xi$ is the sampling wavenumber. Now, using once again the convolution theorem the Fourier transform of the discrete modulated ensemble member becomes

$$
\begin{aligned}
\mathscr{F}\left(x^{(k)} \circ \chi^{(m)} \circ \gamma \circ \mathrm{III}_{d \xi}\right)(\kappa) & =\frac{1}{2 \pi} \mathscr{F}\left(x^{(k)} \circ \chi^{(m)} \circ \gamma\right)^{*} \mathscr{F} \operatorname{III}_{d \xi}(\kappa) \\
& =\frac{1}{d \xi} \sum_{l=-\infty}^{\infty} \int_{-\infty}^{\infty} \mathscr{F}\left(x^{(k)} \circ \chi^{(m)} \circ \gamma\right)\left(\kappa^{\prime}\right) \delta\left(\kappa-l \kappa_{\text {sample }}-\kappa^{\prime}\right) d \kappa^{\prime} \\
& =\frac{1}{d \xi} \sum_{l=-\infty}^{\infty} \mathscr{F}\left(x^{(k)} \circ \chi^{(m)} \circ \gamma\right)\left(\kappa-l \kappa_{\text {sample }}\right) \\
& =\frac{c_{\gamma} L_{\mathrm{MC}}}{2 \pi d \xi} \int_{-\infty}^{\infty}\left[\sum_{l=-\infty}^{\infty} \mathscr{F} x^{(k)}\left(\kappa-\kappa^{\prime}-l \kappa_{\text {sample }}\right)\right] \exp \left(-i \kappa^{\prime} \xi_{m}\right) \operatorname{sinc}\left(\frac{1}{2} L_{\mathrm{MC}} \kappa^{\prime}\right) d \kappa^{\prime}
\end{aligned}
$$


Comparison of (A2) and (A5) shows that discretization gives rise to aliasing: $\mathscr{F} x^{(k)}\left(\kappa-\kappa^{\prime}\right)$ has been replaced by $\sum_{l=-\infty}^{\infty} \mathscr{F} x^{(k)}\left(\kappa-\kappa^{\prime}-l \kappa_{\text {sample }}\right)$. Consequently, for the discrete modulated ensemble member the Fourier transform at wavenumber $\kappa$ is a weighted combination dominated by Fourier components with wavenumbers close to $\kappa$ and wavenumbers differing from $\kappa$ by a multiple of $\kappa_{\text {sample. }}$. In particular, if $x^{(k)}$ is generated using (13) or (17) the discrete modulated ensemble member also has nonzero Fourier components for $|\kappa|>\left|\kappa_{ \pm N_{1 / 2}}\right|$. As a consequence, Fourier components of the discrete modulated ensemble member for $\kappa \sim \kappa_{N_{1 / 2}}\left(\kappa \sim \kappa_{-N_{1 / 2}}\right)$ are not only combinations of the Fourier components of $x^{(k)}$ with wavenumbers near $\lesssim \kappa_{N_{1 / 2}}\left(\geq \kappa_{-N_{1 / 2}}\right)$, but also with wavenumbers near $\kappa_{-N_{1 / 2}}\left(\kappa_{N_{1 / 2}}\right)$.

\section{REFERENCES}

Anderson, J. L., 2007: Exploring the need for localization in ensemble data assimilation using a hierarchical ensemble filter. Physica D, 230, 99-111, doi:10.1016/j.physd.2006.02.011.

_ 2012: Localization and sampling error correction in ensemble Kalman filter data assimilation. Mon. Wea. Rev., 140, 23592371, doi:10.1175/MWR-D-11-00013.1.

Bennett, A. F., 1992: Generalized inverses of dynamical models. Inverse Methods in Physical Oceanography, G. K. Batchelor et al., Eds., Cambridge University Press, 112-150.

Berre, L., 2000: Estimation of synoptic and mesoscale forecast error covariances in a limited-area model. Mon. Wea. Rev., 128, 644-667, doi:10.1175/1520-0493(2000)128<0644: EOSAMF $>2.0 . \mathrm{CO} ; 2$.

Bishop, C. H., and D. Hodyss, 2007: Flow-adaptive moderation of spurious ensemble correlations and its use in ensemble-based data assimilation. Quart. J. Roy. Meteor. Soc., 133, 2029-2044, doi:10.1002/qj.169.

$\longrightarrow$, and - , 2009a: Ensemble covariances adaptively localized with ECO-RAP. Part 1: Tests on simple error models. Tellus, 61A, 84-96, doi:10.1111/j.1600-0870.2008.00371.x.

$\longrightarrow$, and $-2009 \mathrm{~b}$ : Ensemble covariances adaptively localized with ECO-RAP. Part 2: A strategy for the atmosphere. Tellus, 61A, 97-111, doi:10.1111/j.1600-0870.2008.00372.x.

— and _ - 2011: Adaptive ensemble covariance localization in ensemble 4D-VAR state estimation. Mon. Wea. Rev., 139, 1241-1255, doi:10.1175/2010MWR3403.1.

,,-- P. Steinle, H. Sims, A. M. Clayton, A. C. Lorenc, D. M. Barker, and M. Buehner, 2011: Efficient ensemble covariance localization in variational data assimilation. Mon. Wea. Rev., 139, 573-580, doi:10.1175/2010MWR3405.1.

Buehner, M., 2012: Evaluation of a spatial/spectral covariance localization approach for atmospheric data assimilation. Mon. Wea. Rev., 140, 617-636, doi:10.1175/MWR-D-10-05052.1.

— , and M. Charron, 2007: Spectral and spatial localization of background-error correlations for data assimilation. Quart. J. Roy. Meteor. Soc., 133, 615-630, doi:10.1002/qj.50.

— covariance localisation. Tellus, 67A, 28027, doi:10.3402/ tellusa.v67.28027.

— , P. L. Houtekamer, C. Charette, H. L. Mitchell, and B. He, 2010: Intercomparison of variational data assimilation and the ensemble Kalman filter for global deterministic NWP. Part II: One-month experiments with real observations. Mon. Wea. Rev., 138, 1567-1586, doi:10.1175/2009MWR3158.1.

Chelton, D. B., R. A. deSzoeke, M. G. Schlax, K. El Naggar, and N. Siwertz, 1998: Geographical variability of the first baroclinic Rossby radius of deformation. J. Phys. Oceanogr., 28, 433-460, doi:10.1175/1520-0485(1998)028<0433: GVOTFB $>2.0 . \mathrm{CO} ; 2$.

Clayton, A. M., A. C. Lorenc, and D. M. Barker, 2013: Operational implementation of a hybrid ensemble/4D-Var global data assimilation system at the Met Office. Quart. J. Roy. Meteor. Soc., 139, 1445-1461, doi:10.1002/qj.2054.

Courtier, P., 1997: Dual formulation of four-dimensional variational assimilation. Quart. J. Roy. Meteor. Soc., 123, 24492461, doi:10.1002/qj.49712354414.

_ _ J.-N. Thépaut, and A. Hollingsworth, 1994: A strategy for operational implementation of 4D-Var, using an incremental approach. Quart. J. Roy. Meteor. Soc., 120, 1367-1387, doi:10.1002/qj.49712051912.

Deckmyn, A., and L. Berre, 2005: A wavelet approach to representing background error covariances in a limited-area model. Mon. Wea. Rev., 133, 1279-1294, doi:10.1175/MWR2929.1.

Derber, J., and F. Bouttier, 1999: A reformulation of the background error covariance in the ECMWF global data assimilation system. Tellus, 51A, 195-221, doi:10.3402/tellusa.v51i2.12316.

Engelberg, S., Ed., 2007: The Wiener-Khinchin theorem and applications. Random Signals and Noise: A Mathematical Introduction, CRC Press, 125-147.

Gaspari, G., and S. E. Cohn, 1999: Construction of correlation functions in two and three dimensions. Quart. J. Roy. Meteor. Soc., 125, 723-757, doi:10.1002/qj.49712555417.

Gauthier, P., M. Buehner, and L. Fillion, 1998: Backgrounderror statistics modelling in a $3 \mathrm{D}$ variational data assimilation scheme: Estimation and impact on the analyses. Proc. ECMWF Workshop on Diagnosis of Data Assimilation Systems, Reading, United Kingdom, ECMWF, 131-145, https://www.ecmwf.int/sites/default/files/elibrary/1999/9500background-error-statistics-modelling-3d-variational-dataassimilation-scheme-estimation-and.pdf.

Geyer, C. J., 1992: Practical Markov chain Monte Carlo. Stat. Sci., 7, 473-483, doi:10.1214/ss/1177011137.

Hamill, T. M., J. S. Whitaker, and C. Snyder, 2001: Distancedependent filtering of background error covariance estimates in an ensemble Kalman filter. Mon. Wea. Rev., 129, 2776-2790, doi:10.1175/1520-0493(2001)129<2776:DDFOBE > 2.0.CO;2.

Houtekamer, P. L., and H. L. Mitchell, 1998: Data assimilation using an ensemble Kalman filter technique. Mon. Wea. Rev., 126, 796-811, doi:10.1175/1520-0493(1998)126<0796: DAUAEK $>2.0 . \mathrm{CO} ; 2$.

$\longrightarrow$, and - 2001: A sequential ensemble Kalman filter for atmospheric data assimilation. Mon. Wea. Rev., 129, 123-137, doi:10.1175/1520-0493(2001)129<0123:ASEKFF>2.0.CO;2.

Kim, S. Y., P. M. Kosro, and A. L. Kurapov, 2014: Evaluation of directly wind-coherent near-inertial surface currents off Oregon using a statistical parameterization and analytical and numerical models. J. Geophys. Res. Oceans, 119, 6631-6654, doi:10.1002/2014JC010115.

Kirchgessner, P., L. Nerger, and A. Bunse-Gerstner, 2014: On the choice of an optimal localization radius in ensemble Kalman filter methods. Mon. Wea. Rev., 142, 2165-2175, doi:10.1175/ MWR-D-13-00246.1.

Kuhl, D. D., T. E. Rosmond, C. H. Bishop, J. McLay, and N. L. Baker, 2013: Comparison of hybrid ensemble/4DVar 
and 4DVar within the NAVDAS-AR data assimilation framework. Mon. Wea. Rev., 141, 2740-2758, doi:10.1175/ MWR-D-12-00182.1.

Kurapov, A. L., D. Foley, P. T. Strub, G. D. Egbert, and J. S. Allen, 2011: Variational assimilation of satellite observations in a coastal ocean model off Oregon. J. Geophys. Res., 116, C05006, doi:10.1029/2010JC006909.

Liu, Y., P. MacCready, and B. M. Hickey, 2009: Columbia River plume patterns in summer 2004 as revealed by a hindcast coastal ocean circulation model. Geophys. Res. Lett., 36, L02601, doi:10.1029/2008GL036447.

Lorenc, A. C., 2003: The potential of the ensemble Kalman filter for NWP-A comparison with 4D-Var. Quart. J. Roy. Meteor. Soc., 129, 3183-3203, doi:10.1256/qj.02.132.

Mitchell, H. L., P. L. Houtekamer, and G. Pellerin, 2002: Ensemble size, balance, and model-error representation in an ensemble Kalman filter. Mon. Wea. Rev., 130, 2791-2808, doi:10.1175/ 1520-0493(2002)130<2791:ESBAME $>2.0 . C O ; 2$.

Moore, A. M., H. G. Arango, G. Broquet, B. S. Powell, A. T. Weaver, and J. Zavala-Garay, 2011: The Regional Ocean
Modeling System (ROMS) 4-dimensional variational data assimilation systems: Part I-System overview and formulation. Prog. Oceanogr., 91, 34-49, doi:10.1016/j.pocean.2011.05.004.

Ngodock, H., and M. Carrier, 2014: A 4DVAR system for the Navy Coastal Ocean Model. Part I: System description and assimilation of synthetic observations in Monterey Bay. Mon. Wea. Rev., 142, 2085-2107, doi:10.1175/ MWR-D-13-00221.1.

Oppenheim, A. V., and R. W. Schafer, Eds., 1989: Fourier analysis of signals using the discrete Fourier transform. Discrete-Time Signal Processing, Prentice Hall, 695-767.

Oregon State Univerity, 2017: The Oregon Washington coastal ocean forecast system: The interactive viewer. Accessed 6 March 2017, http://ingria.coas.oregonstate.edu/rtdavow/.

Strichartz, R., Ed., 1994: Fourier analysis. A Guide to Distribution Theory and Fourier Transforms, CRC Press, 117-122.

Yu, P., A. L. Kurapov, G. D. Egbert, J. S. Allen, and P. M. Kosro, 2012: Variational assimilation of HF radar surface currents in a coastal ocean model off Oregon. Ocean Modell., 4950, 86104, doi:10.1016/j.ocemod.2012.03.001. 